\title{
AGMON'S COMPLEX TAUBERIAN THEOREM AND CLOSED ORBITS FOR HYPERBOLIC AND GEODESIC FLOWS
}

\author{
MARK POLLICOTT
}

(Communicated by Paul S. Muhly)

\begin{abstract}
In this note we give an alternative proof of Sharp's dynamical analogue of Merten's theorem for hyperbolic flows. Our use of a tauberian theorem of Agmon also allows us to get better error terms.
\end{abstract}

\section{INTRODUCTION}

In an earlier paper, Parry and the author proved that the asymptotic distribution of closed orbits for hyperbolic flows is analogous to the prime number theorem in number theory [4]. More recently, Sharp has derived the corresponding analogues for closed orbits of Merten's theorems on prime numbers [5]. (Essentially, these results on the distribution of prime numbers are weaker but more accessible than the prime number theorem.)

The purpose of this short note is to present an alternative derivation of Sharp's results which has the significant advantage that we are able to obtain far better error estimates.

Let $\phi_{t}: \Lambda \rightarrow \Lambda$ be a (weak-mixing) hyperbolic flow restricted to a basic set $\Lambda$, with topological entropy $h>0$. Let $\tau$ denote a closed orbit of least period $\lambda(\tau)$ and we shall denote $N(\tau)=e^{h \lambda(\tau)}$.

\section{Theorem.}

(i) $\sum_{N(\tau) \leq x} \frac{1}{N(\tau)}=\log \log x+A+o\left(\frac{1}{\log x}\right)$

(ii) $\sum_{N(\tau) \leq x}\left(1-\frac{1}{N(\tau)}\right)=\frac{B}{\log x}\left(1+o\left(\frac{1}{\log x}\right)\right)$,

where the constants $A, B \neq 0$ will be specified later.

Merten's theorems would essentially correspond to replacing $N(\tau)$ by prime numbers in these formulae. Sharp's theorems have the weaker error terms $O(1)$ [5]. The new ingredient in our analysis is a tauberian theorem due to Agmon which, once assumed, leads to a shorter proof with better error estimates.

Received by the editors December 19, 1989.

1980 Mathematics Subject Classification (1985 Revision). Primary 58FXX. 


\section{DEFINITIONS AND PROOFS}

Let $M$ be a compact manifold, and let $\phi_{t}: M \rightarrow M$ be a $C^{1}$ flow. A compact $\phi$-invariant set $\Lambda$ is hyperbolic if $T_{\Lambda} M=E^{0}+E^{s}+E^{u}$ splits into three $D \phi$-invariant bundles satisfying

(a) $E^{0}$ is one-dimensional and tangent to the flow; and

(b) $\exists C, \lambda>0$, such that

$$
\left\|\left.D \phi_{t}\right|_{E^{s}}\right\| \leq C e^{-\lambda t} \text { and }\left\|\left.D \phi_{-t}\right|_{E} u\right\| \leq C e^{-\lambda t}, \quad \forall t \geq 0 .
$$

We call $\Lambda$ a basic set if

(i) The periodic orbits of $\left.\phi\right|_{\Lambda}$ are dense in $\Lambda$;

(ii) $\Lambda$ contains a dense orbit; and

(iii) $\exists$ open set $U \supset \Lambda$ with $\Lambda=\bigcap_{t \in \mathbb{R}} \phi_{t} U$

The classic example of a hyperbolic flow is a geodesic flow on the unit tangent bundle $M=S_{1} V$ of a compact manifold $V$ with negative sectional curvatures (where in this case $\Lambda=M$ ).

We define a zeta function by

$$
\zeta(s)=\prod_{\tau}\left(1-N(\tau)^{-s}\right)^{-1}, \quad s \in \mathbb{C}
$$

(which always converges to a nonzero analytic function on $\operatorname{Re}(s)>1$ ).

We say the flow $\phi_{t}: \Lambda \rightarrow \Lambda$ is (topologically) weak-mixing if $F \phi_{t}=e^{i a t} F$, $a \neq 0, F \in C^{0}(\Lambda)$, has no nontrivial solutions.

Proposition 1. If $\phi_{t}: \Lambda \rightarrow \Lambda$ is weak-mixing, then $\zeta(s)$ has a nonzero analytic extension to a neighborhood of $\operatorname{Re}(s) \geq 1$, except for a simple pole at $s=1$ (cf. [4]).

We can expand the expression for the zeta function, when $\operatorname{Re}(s)>1$ as

$$
\log \zeta(s+1)=\sum_{n=1}^{+\infty} \sum_{\tau} \frac{N(\tau)^{-(s+1) n}}{n}=f_{1}(s)+f_{2}(s)
$$

where

$$
f_{1}(s)=\sum_{\tau} \frac{N(\tau)^{-(s+1) n}}{n} \text { and } f_{2}(s)=\sum_{n=2}^{+\infty} \sum_{\tau} \frac{N(\tau)^{-(s+1) n}}{n} .
$$

The function $f_{2}(s)$ is known to be analytic on $\operatorname{Re}(s)>-\frac{1}{2}$ (cf. [3]) and thus we see from Proposition 1 that the function $f_{1}(s)$ has a nonzero analytic extension to a neighborhood of $\operatorname{Re}(s) \geq 0$, except for a singularity at $s=0$ of the form $\log \left(\frac{1}{s}\right)$. as

If we introduce $\alpha(u)=\sum_{N(\tau) \leq e^{u}} \frac{1}{N(\tau)}, u>0$, then we can re-express $f_{1}(s)$

$$
f_{1}(s)=\int_{0}^{\infty} e^{s u} d \alpha(u)
$$

The above observations, combined with Proposition 1, allow us to write

$$
\int_{0}^{\infty} e^{-s u} d \alpha(u)+f_{2}(s)=\log \left(\frac{1}{s}\right)+\chi(s),
$$


where $\chi(s)$ is analytic for $\operatorname{Re}(s) \geq 0$. In particular, $\chi(0)$ is the logarithm of the residue $\operatorname{res}(\zeta)$ for $\zeta(s)$ at $s=1$.

We can derive the following tauberian theorem from the more general results of Agmon:

Proposition 2. Let $\alpha(u)$ be real and nondecreasing, and define a function $F(s)$ $=\int_{0}^{\infty} e^{-s u} d \alpha(u)$ on $\operatorname{Re}(s)>0$. Assume that

(a) the singularities for $F(s)$ on the imaginary axis are contained in the set $\{2 \pi i k \mid k \in \mathbb{Z}\} ;$ and

(b) $F(s)-\log \left(\frac{1}{s}\right)$ is analytic at $s=0$.

Then $\alpha(u)=\log u+A+o\left(\frac{1}{u}\right)$, where

$$
A=\lim _{s \rightarrow 0^{+}}\left[F(s)-\int_{1}^{\infty} \frac{e^{-s u}}{u} d u\right]
$$

(cf. [2, Theorem 7.1 and p. 480]).

We can apply Proposition 2, with the choice $F=f_{1}$, to (1.2). By substituting $u=\log x$ we deduce part (i) of the Theorem as follows.

To calculate $A$, we can use the standard exponential integral expansion:

$$
\int_{1}^{\infty} \frac{e^{-s u}}{u} d u=-\gamma-\log (s)-\sum_{n=1}^{\infty} \frac{(-s)^{n}}{n \cdot n !} \quad(s>0),
$$

where $\gamma$ is Euler's constant (cf. ([1, p. 472 and p. 476]). By substituting into the formula for $A$ in the above proposition and then applying to (1.2) we get

$$
\begin{aligned}
A & =\lim _{s \rightarrow 0^{+}}\left[f_{1}(s)-\int_{1}^{\infty} \frac{e^{-s u}}{u} d u\right] \\
& =\lim _{s \rightarrow 0^{+}}\left[\left(\log \left(\frac{1}{s}\right)-f_{2}(s)+\log \operatorname{res}(\zeta)\right)+(\gamma+\operatorname{logs})\right] \\
& =\log \operatorname{res}(\zeta)-f_{2}(0)+\gamma .
\end{aligned}
$$

This completes the proof of part (i) of the theorem.

We shall now proceed to the proof of part (ii) of the theorem.

Let $\beta(x)=\sum_{N(\tau) \leq x} \log \left(1-\frac{1}{N(\tau)}\right)$, for $x>0$. Then we can write

$$
\begin{aligned}
\alpha(x)+\beta(x) & =\sum_{N(\tau) \leq x}\left\{\frac{1}{N(\tau)}+\log \left(1-\frac{1}{N(\tau)}\right)\right\} \\
& =\sum_{N(\tau) \leq x} \sum_{n=2}^{+\infty} \frac{-1}{N(\tau)^{n}} \rightarrow-f_{2}(0), \quad \text { as } x \rightarrow+\infty
\end{aligned}
$$

We can estimate the rate of convergence in (1.3) by

$$
\begin{aligned}
\sum_{N(\tau) \geq x} \sum_{n=2}^{+\infty} \frac{1}{N(\tau)^{n}} & =\sum_{N(\tau) \geq x} \frac{1}{N(\tau)^{2}(1-1 / N(\tau))} \\
& =O\left(\int_{u}^{+\infty} \frac{\alpha(y)}{y^{2}} d y\right)=O\left(\frac{1}{x^{\alpha}}\right),
\end{aligned}
$$

for any $0<\alpha<1$, by part (i). 
From (1.3), (1.4), and part (i) of the theorem,

$$
\beta(x)=-\log \log x-\log \operatorname{res}(\zeta)-\gamma+o\left(\frac{1}{\log x}\right) .
$$

Finally, we observe that $\beta(x)=\log \left[\prod_{N(\tau) \leq x}\left(1-\frac{1}{N(\tau)}\right)\right]$, and so (1.5) gives the estimate for part (ii) of the Theorem. In particular, we observe that $B=$ $e^{-\gamma} / \operatorname{res}(\zeta)$. This completes the proof of part (ii) of the theorem.

Remark. Similar results are true in the cases other than weak-mixing, except that we must replace $o\left(\frac{1}{\log x}\right)$ by $O\left(\frac{1}{\log x}\right)$. The asymptotic formula for $\alpha(x)=$ $\sum_{N(\tau) \leq x} \frac{1}{N(\tau)}$ then comes from direct computation. More specifically, the flow corresponds to a suspension by a function with constant value $c$ over a subshift of finite type with transition matrix $A$, say; then $\alpha(x)=\sum_{e^{c k} \leq x}\left(\operatorname{trace} A^{k} / e^{c k \cdot k}\right)$, and the estimate becomes a simple calculation.

Part (ii) of the theorem follows as before, with obvious changes.

Remark. In the case of geodesic flow on a compact surface of constant curvature $-1, \operatorname{res}(\zeta)$ is known to be intimately related to the functional determinant of the Laplacian.

\section{Generalizations}

The method of proof works in more general settings, with trivial modifications, provided we have an analogue of Proposition 1 for the corresponding zeta function. We give below two examples:

(i) If we consider a finite Galois $G$-covering flow $\tilde{\phi}_{t}: \widetilde{\Lambda} \rightarrow \widetilde{\Lambda}$ for $\phi_{t}: \Lambda \rightarrow$ $\Lambda$ when we can fix a conjugacy class $C$ in $G$, clearly, $\sum_{N(\tau) \leq x}\langle\tau\rangle=C \frac{1}{N(\tau)}=$

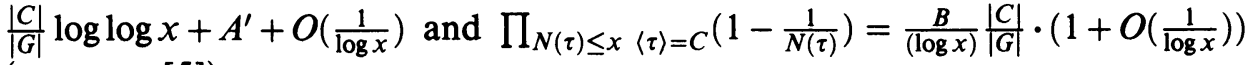
(compare [5]).

(ii) We can consider the geodesic flow on the (non-compact) modular surface. The analogue of Proposition 1 is known [6], and the proof of the theorem goes through as before.

\section{REFERENCES}

1. G. Arfken, Mathematical methods for physicists, Academic Press, New York, 1970.

2. S. Agmon, Complex variable tauberians, Trans. Amer. Math. Soc. 74 (1953), 444-481.

3. W. Parry, Bowen's equidistribution theory and the Dirichlet density theorem, Ergodic Theory Dynamical Systems 4 (1984), 117-134.

4. W. Parry and M. Pollicott, An analogue of the prime orbit theorem for closed orbits of Axiom A flows, Ann. of Math. 118 (1983), 573-591.

5. R. Sharp, An analogue of Mertens' theorem for closed orbits of Axiom A flows, preprint, Warwick University.

6. A. Verkov, Spectral theory of automorphic functions, the Selberg zeta function, and some problems of analytic number theory, Russian Math. Surv. 34 (1979), 79-153.

Department of Mathematics, University of Porto, Praça Gomes Teixeira, 4000 Porto, Portugal 